\title{
Effects of External Application of Ginkgo Semen Paste on Tinea Corporis Model of Rat
}

\author{
Yan LI, Ming BAI, Ting WANG, Dandan LIU, Mingsan MIAO* \\ Henan University of Traditional Chinese Medicine, Henan, 450008, China
}

\begin{abstract}
Objective: To investigate the effects of external application of Ginkgo Semen (GS) paste on tinea corporis model of rat. Methods: Tinea corporis rats model were established by vaccination trichophyton mentagrophytes in skin wound. Observe the effects of the high, low dose of GS alcohol paste group and the high, low of GS water paste on symptoms integral before and after treatment, the skin lesions inverse culture and the local tissue morphology in tinea corporis rats model. Results: Tinea ulcer model rats successfully. Compared with the model group, the high, low dose of GS alcohol paste group and the high, low GS water paste could significantly or remarkably reduce the symptoms integral, remarkably increase the skin inverse culture turn rate and improve the pathological changes. Conclusion: The external application of GS paste has remarkable curative effect on tinea corporis rats model.
\end{abstract}

KEYWORD: Ginkgo Semen(GS) paste; External application; Tinea corporis model

\section{INTRODUCTION}

GS have convergence lung, given Asthma, only with cloud and reduce it's effectiveness [1]. Its medicinal properties of high value, which is TCM Medicinal and Edible. Its resistance value is very high and contained flavonoids, rich antibacterial protein, bilobalide and other components [2]. <Materia Medica summary>says: " power post astringent cooked food warm the feiqi, given sputum Asthma, Asthma convergence, reduce it, only with muddy, insecticide to lice " [3]. Through this experiment to observe its effect on rat tinea corporis model, verified of GS external with insecticidal power and standardize its external function.

\section{MATERIALS}

\subsection{Animals}

80Wistar rats, male and female, weighted 180-220g, supplied by the Experimental Animal Center of Heben Medical. Animal permit number: 1103158.

\subsection{The experimental instrument and drugs}

AR1140/C electronic analytical balance, Ohaus (Shanghai) Company; MJ-180B -type mold incubator, Shanghai Yuejin Medical Instrument Production; GS was identified by the department of Henan University of Traditional Chinese Medicine chamber for seeds of Gink-go biloba L..GS paste preparation method: GS medicinal powder, through the 120 mesh sieve. Transfered paste by water or alcohol: High dose of $0.60 \mathrm{~g} \cdot \mathrm{ml}^{-1}$, low dose of $0.30 \mathrm{~g} \cdot \mathrm{ml}^{-1}$; The matrix group is $60 \%$ alcohol. Strains is the Trichophyton mentagrophytes standard strain, whijh purchased from Chinese Academy of Medical Sciences and Institute of Dermatology Prevention Culture Collection, bacteria No. CMCC1F15a; Potato dextrose agar, Shanghai Yuanye Biological Technology Co.,Ltd. production; Xianyaogao, Heilongjiang Tianlong Pharmaceutical Co.,Ltd production; Qiaomier creams, Guangzhou Yibai Yahua Co., Ltd. production .

\section{METHODS}

\subsection{Preparation of culture medium and bacterial liquid}

Take potato peel and cut into small piece with $200 \mathrm{~g}$ and into1000 $\mathrm{ml}$ of distilled water Zhulan, filtered with gauze, plus glucose $20 \mathrm{~g}$, agar $15 \mathrm{~g}$, heating and stirring evenly, after cooling slightly to make the $1000 \mathrm{ml}$, boil until all dissolved by $121{ }^{\circ} \mathrm{C}, 15 \mathrm{~min}$ high pressure sterilization, cooled to $55{ }^{\circ} \mathrm{C}$ after sterilization and poured vessel spare. Take the Trichophyton mentagrophytes on the potato dextrose agar medium inoculated with fungal culture, $10 \mathrm{~d}$ culture medium $26{ }^{\circ} \mathrm{C}$, removed the colony sterile Sodium Chloride Injection after grinding to made 
with strain $10^{7} \cdot \mathrm{ml}^{-1}$ of bacteria suspension.

\subsection{Build the tinea corporis model of rat and observation index [4]}

Before one day of the experiment would each rat right back hair removed, which covered an area of 9 $\mathrm{cm}^{2}$.Then shed coated hair removal liquid lightly, after 8min with warm water to wash hair removal liquid residue. The rats after depilated were randomly selected 10 rats as the control group, the remained 70 rats in the shed at the skin with a scalpel scratch until blood oozing for the degree. Then take the bacteria culture, smeared on the wound with a razor blade, wrapped with gauze and then tape wrapped to prevent break. Bacterial contamination observed daily and repeat until the 9th day lesions appeared. After contaminated successfully, according to the literature method severity score [5]. (No lesions were "0", a few erythematous papules or lesions tend to heal, grow new hair were "1", an island like erythema fusion or scattered around and red were "2", a comprehensive expansion of a large number of erythema, scales and thick skin were"3", accompanied by bleeding very lesions were "4"). According to the lesion score and male and female the rats were randomly principle divided into 7 groups, Topical respectively high and low dose of GS alcohol paste $\left(0.60 \mathrm{~g} \cdot \mathrm{ml}^{-1}\right.$, $\left.0.30 \mathrm{~g} \cdot \mathrm{ml}^{-1}\right)$, high and low dose of GS water paste $\left(0.60 \mathrm{~g} \cdot \mathrm{ml}^{-1}, 0.30 \mathrm{~g} \cdot \mathrm{ml}^{-1}\right)$, Xianyaogao (thickness of $2 \mathrm{~mm}$ ), the control group and the model group smeared $0.9 \%$ sodium chloride injection of $1.0 \mathrm{ml}$, the excipient matrix group paste coated (thickness of $2 \mathrm{~mm}$ ). The rats were used two layers of gauze dressed liquid adsorption corresponding parts of the back sides of tinea, treated and covered with plastic wrap, after administration of $6 \mathrm{~h}$, used the $0.9 \%$ sodium Chloride Injection to cleaned the partial. Administered once a day, medicated for 12 days continuously.

Rats were observed healing lesions daily, every four days reference standard severity scores were scored and recorded, 12th day after administrated of $6 \mathrm{~h}$ drug and saline washed. The rats were killed, taking parts of the skin lesions, lesions at each per rat take 4 skin, which a skin wound fixed in $10 \%$ formalin solution, skin inflammation was observed under paraffin-embedded sections, HE stained and microscopied. The remained three into $70 \%$ soaked ethanol for 2-3 minutes, bacteria was killed, after washed with sterile saline in a sterile room inoculated in potato dextrose agar medium and marked and then put the dish in the incubator $37{ }^{\circ} \mathrm{C}$ culture, removed after 1 week, required to observe the growth of Trichophyton ringworm.

\subsection{Methods Statistical Analysis}

Data analysis used SPSS 17.0 for windows for statistical treatment. measurement data represented by mean \pm variance ( $x \pm s$ ), group comparison used analysis of variance; ranked data used Ridit test.

\section{RESULTS}

\subsection{Impact on symptom score on the tinea corporis model of rat See table 1.}

From table 1, significantly higher the symptoms of tinea integra on $1,4,8,12$ day of each group were compared with the blank group $(P<0.01)$, indicating the model was successful. Compared with the blank group, the symptoms integral of other groups don't have marked disparity on first day $(P>0.05)$, which demonstrate that the grouping is uniform; In forth day ,high dose of GS alcohol paste group and Xianyaogao group could remarkably reduce the symptoms of tinea integral $(P<0.01)$, Low dose of GS alcohol paste group and high of GS water paste group could obviously reduce the symptoms of tinea integral $(P<0.05)$; In 8th day, high, low dose of GS alcohol paste group and Xianyaogao group could remarkably reduce the symptoms of tinea integral $(P<0.01)$, high of GS water paste group could obviously reduce the symptoms of tinea integral $(P<0.05)$; In12th day, high, low dose of GS alcohol paste group and high, low of GS water paste group could remarkably reduce the symptoms of tinea integral $(P<0.01)$.

Table 1. Impact on before and after the symptom score in the tinea corporis model of rat $(\bar{X} \pm S)$

\begin{tabular}{|c|c|c|c|c|c|}
\hline \multirow{2}{*}{ Group } & \multirow{2}{*}{$\mathrm{n}$} & \multicolumn{3}{|c|}{ The rat caused by Tinea Corporis on symptoms integral at different time } \\
\cline { 3 - 6 } & & $1 \mathrm{~d}$ & $4 \mathrm{~d}$ & $8 \mathrm{~d}$ & $12 \mathrm{~d}$ \\
\hline Blank group & 10 & $0.0 \pm 0.0^{* *}$ & $0.0 \pm 0.0^{* *}$ & $0.0 \pm 0.0^{* *}$ & $0.0 \pm 0.0^{* *}$ \\
\hline Model group & 10 & $3.4 \pm 0.5$ & $3.2 \pm 0.4$ & $2.7 \pm 0.5$ & $2.1 \pm 0.3$ \\
\hline Matrix group & 10 & $3.5 \pm 0.5$ & $3.1 \pm 0.3$ & $2.6 \pm 0.5$ & $1.9 \pm 0.3$ \\
\hline Xianyaogao group & 10 & $3.4 \pm 0.5$ & $2.7 \pm 0.5^{* *}$ & $1.8 \pm 0.4^{* *}$ & $0.7 \pm 0.5^{* *}$ \\
\hline High dose of GS alcohol paste group & 10 & $3.4 \pm 0.5$ & $2.7 \pm 0.5^{* *}$ & $1.9 \pm 0.3^{* *}$ & $0.8 \pm 0.4^{* *}$ \\
\hline Low dose of GS alcohol paste group & 10 & $3.4 \pm 0.5$ & $2.8 \pm 0.4^{*}$ & $2.0 \pm 0.5^{* *}$ & $1.2 \pm 0.4^{* *}$ \\
\hline High of GS water paste group & 10 & $3.5 \pm 0.5$ & $2.8 \pm 0.4^{*}$ & $2.2 \pm 0.5^{*}$ & $1.5 \pm 0.5^{* *}$ \\
\hline Low of GS water paste group & 10 & $3.5 \pm 0.5$ & $2.9 \pm 0.3$ & $2.3 \pm 0.7$ & $1.4 \pm 0.5^{* *}$ \\
\hline
\end{tabular}

Note: Compared with the model group, $* P<0.05, * * P<0.01$ 


\subsection{Impact on topical skin lesions inverse cultured on the tinea corporis model of rat See table 2.}

From table 2 , by $\chi^{2}$ analysis. Compared with the model group, the negative rate of topical skin lesions inverse cultured of matrix group don't have marked disparity $(P>0.05)$; Each dose of GS alcohol paste group each dose of GS water paste group and Xianyaogao group could remarkably increase the negative rate of topical skin lesions inverse cultured $(P<0.01)$.

Table 2. Effect of GS paste topical skin lesions inverse cultured on tinea corporis model of rat

\begin{tabular}{|c|c|c|c|c|c|}
\hline Group & $\mathrm{N}$ & Negative & Positive & Total & The negative rate (\%) \\
\hline Model group & 10 & 7 & 23 & 30 & 23.33 \\
\hline Matrix group & 10 & 9 & 21 & 30 & 30.00 \\
\hline Xianyaogao group & 10 & 23 & 7 & 30 & $76.67^{* *}$ \\
\hline High dose of GS alcohol paste group & 10 & 23 & 7 & 30 & $76.67^{* *}$ \\
\hline Low dose of GS alcohol paste group & 10 & 19 & 11 & 30 & $63.33^{* *}$ \\
\hline High of GS water paste group & 10 & 16 & 14 & 30 & $53.33^{* *}$ \\
\hline Low of GS water paste group & 10 & 16 & 14 & 30 & $53.33^{* *}$ \\
\hline
\end{tabular}

Note: Compared with the model group, $* P<0.05$, $* * P<0.01$

\subsection{Influence on pathological changes of rat model of Tinea Corporis See table 3, Figure 1.}

From table 2, by Ridit test analysis. Compared with the blank group, the pathological change of symptoms of tinea pathological lesions in model group was most remarkable $(P<0.01)$, shows that the model copied successfully. Compared with the model group, high, low doses of GS alcohol paste group, high dose of GS water paste group and Xianyaogao group could remarkably reduce the pathological change of symptoms of tinea pathological lesions $(P<0.01)$, low dose of GS water paste group could obviously reduce the pathological change of symptoms of tinea pathological lesions $(P<0.05)$

Table 3. Effect of GS paste on pathological changes of rat model of Tinea Corporis

\begin{tabular}{|l|c|c|c|c|c|c|}
\hline Group & $\mathrm{N}$ & - & + & ++ & +++ & $P$ \\
\hline Blank group & 10 & 10 & 0 & 0 & 0 & $* *$ \\
\hline Model group & 10 & 0 & 0 & 1 & 9 & \\
\hline Matrix group & 10 & 0 & 0 & 4 & 6 & $*$ \\
\hline Xianyaogao group & 10 & 0 & 3 & 7 & 0 & $* *$ \\
\hline $\begin{array}{l}\text { High dose of GS alcohol } \\
\text { paste group }\end{array}$ & 10 & 2 & 5 & 3 & 0 & $* *$ \\
\hline $\begin{array}{l}\text { Low dose of GS alcohol } \\
\text { paste group }\end{array}$ & 10 & 0 & 4 & 6 & 0 & $* *$ \\
\hline $\begin{array}{l}\text { High of GS water paste } \\
\text { group }\end{array}$ & 10 & 4 & 6 & 0 & 0 & $* *$ \\
\hline $\begin{array}{l}\text { Low of GS water paste } \\
\text { group }\end{array}$ & 10 & 1 & 4 & 5 & 0 & $*$ \\
\hline
\end{tabular}

Note:"-" skin epithelial tissue integrity, subcutaneous tissue and hair follicles and other appendages were normal; "+" skin squamous epithelium mild hyperplasia , covered with a amount of inflammatory cell, hair follicles and other adnexal normal;"++" skin epithelial squamous epithelial tissue proliferation, inflammatory cells coated with thin, hair follicles and other adnexal damaged;"+++" skin epithelial squamous epithelial tissue proliferation, inflammatory cell layer coated with thick, hair follicles and other adnexal injury.

Compared with the model group, $* P<0.05, * * P<0.01$

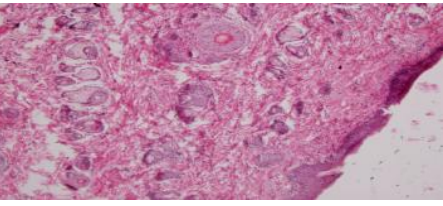

A
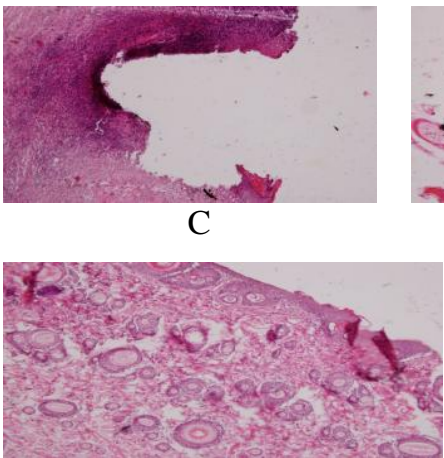

$\mathrm{E}$

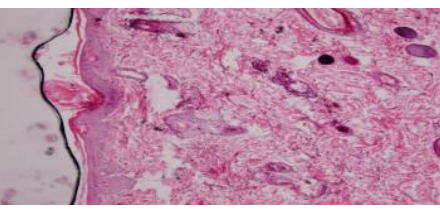

G

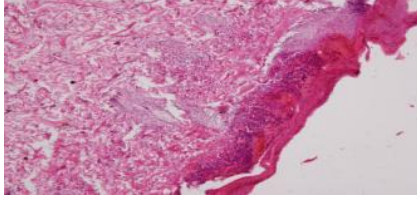

B
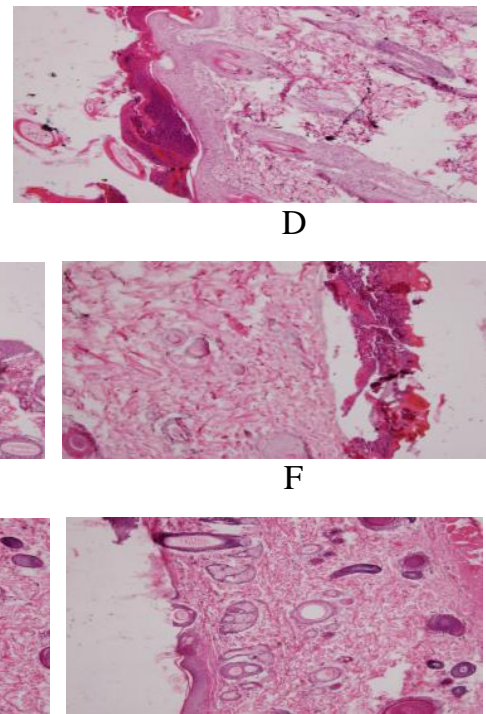

$\mathrm{H}$
A Blank group; B Model group; C Matrix group; D Xianyaogao group; E High dose of GS alcohol paste group; F Low dose of GS alcohol paste grou; G High of GS water paste group; H Low of GS water paste group

Figure 1. Effects of external application of Ginkgo Semen paste on tinea corporis model of rat of the pathological change of symptoms of tinea $(\mathrm{HE} \times 100)$

\section{DISCUSSION}

Tinea corporis caused by epidermal dermatophytes infection fungal skin disease, also known as "round ringworm" or "ringworm", is the clinical common disease and frequently occurring, which caused by hair Trichophyton, Microsporum or Epidermophyton [6]. Trichophyton mentagrophytes fas pro-animal dermatophytes, severe inflammatory response after 
infection, jock itch and ringworm of the body of the second pathogen, after Trichophyton rubrum [7]. Therefore, this experiment adopted Trichophyton mentagrophytes strains for the production of tinea skin wound infection model, the building method is relatively simple and easy to operate.

To better judged the efficacy of GS external of tinea corporis, this experiment used degree index commonly used in research of antifungal drugs symptoms of tinea integra, skin lesions inverse cultured and skin management training sliced to observe the effect of antifungal drug [8]. Before and after the symptoms of tinea integra, can be more directly and scientifically reflect the impact of drugs on the degree of tinea corporis disease; Observe the focus part of the skin if growth of the Trichophyton, with skin lesions inverse culture must be negative growth of Trichophyton tinea strains as judged by the negative rate of topical skin lesions as efficacy index, can from the microscopic point of view further direct reaction of drug efficacy; At the same time the the pathological change of tinea pathological lesions can reflect the degree of inflammatory cells infiltration of the damaged tissue of skin and damaged tissue healing, more directly reflect the efficacy of the drug. The result of experiment show that, in 8th, 12th day, high ,low dose of GS alcohol paste group could significantly reduce the symptoms of tinea integral, remarkably increase the negative rate of topical skin lesions inverse cultured, remarkably reduce the pathological change of symptoms of tinea pathological lesions and reduce inflammatory cell infiltration; In 12th day, high, low dose of GS water paste group could significantly reduce the symptoms of tinea integral, remarkably increase the negative rate of topical skin lesions inverse cultured, remarkably reduce the pathological change of symptoms of tinea pathological lesions; But change of symptoms of tinea pathological lesions in matrix and model groups were the same basically, indicated that vehicle did not affect the mechanism of tinea corporis, but can be modulated drug excipient, easy fixed, can increase the water content of the stratum corneum and transdermal drug absorption capacity [9]. It is suggested that external application of Ginkgo Semen paste on tinea corporis model of rat, which has a good therapeutic effect,especially the high and low doses of GS alcohol paste group.

TCM believes that "tinea" is the Department of fat body phlegm, which caused by the wind, wet, hot, insect invasion of the skin. <Depeibencao>, $<$ Bencaofenjing> record the basic consistently, "this is a rheumatic disease". "Practice used, profit lung, asthma and narrow, check with turbidity. Born used, phlegm reduction, disinfection kill insect"[10]. Modern pharmacological studies had shown that the GS has anti-inflammatory, anti-bacterial, anti- allergic, anti-viral, anti-cancer, dewormed, insecticided and other effect [11]. This study provided experimental support for the external function of GS on insecticidal topical feature and provided a simple and effective treatment method for clinical treatment of tinea corporis.

\section{ACKNOWLEDGEMENTS}

The research work is supported by the National Science and Technology Pillar Program during the 'Eleventh Five-year' Plan Period (No.2008BAI53B09), Science and Technology Innovation Team Plan of Zhengzhou city (No.131PCXTD612).

\section{REFERENCES}

[1] Zhao CF, Zhu LX. Progress of the research extraction and the effect of active components of Ginkgo. Journal of Zhongkai University of Agriculture and Technology, 2009, 22(4):67 -70.

[2] Geng JZ. Study on Optimization of extraction process of ginkgo acid sarcotestas of Ginkgo biloba. Northern Horticulture, 2012(13): 42-44.

[3] Zheng J,Zhang K.Research on Materia of Toxic traditional Chinese medicine of Ginkgo Semen. Shandong Journal of Traditional Chinese Medicine, 2012, 31(7):517-518.

[4] Tang PP, Miao MS. Effects of external application of Daphne genkwa on tinea corporis model ofguinea pig and rat.China Journal of Traditional Chinese Medicine and Pharmacy, 2014, 29(3):722-726.

[5] Zeng Y, Meng XL, Zhou BQ. Effect the antifungal of anti inflammation and anti ringworm cream. Journal of Chengdu Unversity of Tarditional Chinese Medicine, 2005, 28(2):52-53.

[6] Zheng Y, Liu WZ. Research on the treatment of tinea corporis tinea with external treatment of TCM. Chinese Medicine Modern Distance Education of China, 2011, 9(12): 168-169.

[7] Miao MS, Cheng BL, Chen YP. Bitter almond oil puste external influence on mice guinea pig model of prurius. Moder Engineering Solution for the Industry, 2014; (4):1343

[8] Gao LL, Wang GZ, Ge HF, et al. Tinea corporis and tinea cauris in Qingdao: An aetiological inspection. Acta Academiae Medicinae Qingdao Universitatis, 2012, 48(2): 157-158.

[9] Zhang JX, Zhao Y, Gao SZ, et al. Analysis of umbilical therapy in the treatment of diarrhea in children excipient drug modulation. Shandong Journal of Traditional Chinese Medicine, 2012, 48(2):157-158.

[10] Wang JJ, Yang XH, Shen YH, et al. Comparison and analysis of different doses of ginkgo episperm molluscicidal effects of crude preparations. Journal of Chizhou College, 2011, 25(3):31-33.

[11] Zhang HM.T he chemical constituents and pharmacological effects of natural drug of Ginkgo biloba. Journal of Capital Normal University (Natural Science Edition), 2014, 35(3):41-45. 